East African Medical Journal Vol. 83 No. 8 August 2006

SONOGRAPHY AND RISK FACTORS FOR LOWER LIMB DEEP.VENOUS THROMBOSIS AT MULAGO HOSPITAL, UGANDA

F. Mangeni, MBChB, MMed (Rad), Medical Officer, Special Grade, Mulago Hospital, M.G. Kawooya, MBChB, MMed (Rad), Associate Professor, E. Kiguli-Malwadde, MBChB, MMed (Rad), Senior Lecturer, Faculty of Medicine, Makerere University and F. Ssali, MBChB, MMed (Int. Med), Consultant Physician, Mulago Hospital, P.O. Box 7051, Kampala, Uganda

Request for reprints to: Dr. E. Kiguli-Malwadde, Department of Radiology, Mulago Hospital, P.O. Box 7051, Kampala, Uganda

\title{
SONOGRAPHY AND RISK FACTORS FOR LOWER LIMB DEEP VENOUS THROMBOSIS AT MULAGO HOSPITAL,UGANDA
}

\author{
F. MANGENI, M. G. KAWOOYA, E. KIGULI-MALWADDE and F. SSALI
}

\begin{abstract}
Background: Lower limb deep venous thrombosis (DVT), and its sequelae (lower limb chronic venous insufficiency and pulmonary embolism) are now well acknowledged as major haematological problems in the world, for which appropriate and accurate means of diagnosis is necessary. Developments in ultrasound have made it the imaging modality of choice in the diagnosis of lower limb DVT.

Objectives: To determine the sonographic pattern, and identify the risk factors of lower limb DVT. Design: Cross sectional, descriptive study carried out between April 2002 and March 2003.

Setting: Mulago Hospital, Uganda.

Subjects: Eighty six consecutive patients ( 92 limbs), with clinically suspected DVT, were studied by duplex sonography after a thorough risk factor evaluation.

Results: Out of a total of 86 patients clinically suspected to have lower limb DVT, 38(44.2\%) were found to have DVT after sonography. The gender incidence was similar. The left limb was affected in $60 \%$ of cases and the right in $40 \%$. Bilateral DVT was noted in two patients. Most of the patients had acute and extensive DVT.

Conclusion: Duplex ultrasonography is a very useful modality for assessing lower limb DVT, even in a low resource country like Uganda. It demonstrates the wealth of information obtained from sonography.
\end{abstract}

\section{INTRODUCTION}

Deep venous thrombosis (DVT) is part of the spectrum of thromboembolic disease, which also includes pulmonary embolism (PE). Lower limb DVT is potentially dangerous because it causes the majority of $\mathrm{PE}$ and may lead to chronic venous insufficiency of the lower limb veins.

Clinical diagnosis of DVT has been reported to be unreliable $(1,2)$. Contrast venography $(\mathrm{CV})$, has been the mainstay of diagnosis despite its disadvantages, which include invasiveness, exposure to radiation and adverse reactions to contrast agent. DVT may also complicate CV. Contrast studies are also costly compared to ultrasound. Ultrasonography (US) has now become the first line imaging modality because of its advantages i.e. safety, availability, low cost, ability to detect other pathology other than DVT and ability to directly visualise a thrombus. The availability of a US machine with a Doppler component now makes it possible to utilise Grey scale, spectral Doppler and colour flow imaging.

The Mulago Hospital annual records show that 15 to 20 patients are clinically suspected of DVT each month. Objective diagnosis in the past has been by 
$\mathrm{CV}$ and many have been anticoagulated on clinical grounds alone. US, with proven sensitivity of more than $95 \%$ and specificity of more than $95 \%$ has been underutilised at Mulago Hospital.

Data on the prevalence of risk factors of DVT and distribution of thrombi among the lower limb deep veins is lacking at Mulago Hospital.

The study objectives were to establish the prevalence of DVT risk factors among patients with lower limb DVT at Mulago National Referral Hospital, Kampala, Uganda and also to relate the risk factors and clinical features with sonographic findings. The study also aimed at documenting the proportion of patients with ileofemoral, popliteal and calf DVT. This would increase the utilisation of US in the diagnosis of DVT.

\section{MATERIALS AND METHODS}

Between May 2002 and March 2003 in Mulago Hospital, Kampala, Uganda, ninety two limbs of 86 patients with suspected DVT on the basis of clinical examination were studied with Grey scale US with application of venous compression. Doppler US was also employed.

Mulago hospital serves as a national referral hospital for Uganda with a population of about 22 million inhabitants (Uganda population and housing census, September 2002). It is also the teaching hospital for the largest medical school in the country. It is the biggest hospital nationally in terms of bed capacity and occupancy rates, variety of disease conditions and patterns seen and treated, and number of health professionals.

All patients of any age with clinical features of lower limb deep venous thrombosis but with no other associated conditions like fractures, were studied after informed consent had been obtained. They were assured of total anonymity and confidentiality of information divulged in the course of the interviews.

All patients had a duplex US examination done after clinical evaluation. The clinical evaluation included history suggestive of DVT and pertinent to risk factors of DVT. A re-scan after a week was done for all patients whose initial study was negative. A medical officer with a scanning experience of three years and two experienced (more than ten years of US scanning) senior radiologists did the examinations. Thrombosis was diagnosed if the vein was not completely compressible by the transducer or if there was direct visualisation of the clot. Other signs included absence of spontaneous Doppler signals, lack of phasic variation of Doppler signals with respiration and absence of augmentation of venous flow on distal compression. The widest diameters of the thrombosed veins were measured and compared with the normal side where necessary.

All patients were scanned while lying flat on a couch, in supine for the thigh veins and prone or decubitus position for the popliteal and calf veins. Unilateral and bilateral studies were done for patients with unilateral and bilateral symptoms respectively. The entire venous system was imaged systematically using a linear 7.5-10 $\mathrm{MHz}$ transducer of an ATL 1500 US machine (manufactured in 2000). Duplex scans were done. Hard copy sonograms for each scan were obtained for off-line interpretation and record. The information was recorded on precoded data forms.

The data were then analysed using EPI-INFO version 6.0 data analysis package. For two categorical variables, both chi-square tests and odds ratio were used in testing for statistical significance. For continuous variables a student $t$-test was applied with a $p$-value of $<0.05$ considered significant where applicable.

\section{RESULTS}

Socio-demographic profiles: During the period of study 86 subjects ( 92 lower limbs) were recruited. Half of these study patients were outpatients and the rest inpatients. There were 53 females and 33 males whose ages ranged from 12 to 81 years with a mean of 42.9 years.

Thirty eight $(48 \%)$ of the 86 patients had DVT proven by sonography using the earlier mentioned diagnostic criterion. Two patients had bilateral DVT. The age range for those who had DVT was 16 to 73 years with a mean of 42.5 years. Two age peaks at which DVT occurred were observed; 21 to 30 and 61 to 71 years. The male: female ratio of patients with DVT was 1:1.

Risk factors: A number of acquired risk factors for DVT were assessed. A known risk factor was noted in $73.7 \%$ of DVT cases. Immobility or prolonged bed rest was the most prevalent, seen in $52.6 \%$ of DVT cases followed by previous history of DVT $(13.2 \%)$. Other risk factors encountered included; major 
trauma and surgery $(10.5 \%)$, pregnancy and contraceptive pill use (7.9\%) and malignancy and cardiac disease $(5.3 \%$ each).

Clinical features: Limb swelling, pain and oedema constituted the most common clinical features. These features were seen in $97.5 \%, 87.5 \%$ and $90.0 \%$ respectively in patients with positive sonography for DVT.

A comparative increase in the mid-calf circumference of $2 \mathrm{~cm}$ or more was the most reliable sign noted in $31(81.6 \%)$ of DVT cases and in only $39.6 \%$ of negative studies. Homans' sign was unreliable being positive in only $37.5 \%$ of DVT cases.

Sonographic findings: Sixty percent of thrombi were found in the left limb. Most of the patients had multiple vein involvement, which was noted in more than $80 \%$ of cases. Forty two percent of positive cases had DVT extending from femoral to popliteal vein. The common femoral was the vein most involved $(80 \%)$ followed by the superficial femoral $(77.5 \%)$. The deep femoral was the least involved vein $(57.5 \%$ ) among the proximal Jower limb veins. Isolated calf vein DVT was seen in four patients.

The majority of clots had dilated the veins $(90.6 \%$ of clots in the common femoral vein). Fifty one percent of clots were found to be free-floating with partial attachment to the vein (Figures 1 and 2 ). This implies most clots were acute.

Other findings at sonography included;

\section{Figure 1}

A 48-year-old female presented with unilateral swelling of the lower limb. Venous sonography revealed a dilated left common femoral vein (LCFV) with an isoechoeic thrombus extending into the greater sepheenous vein (GSV). The thrombus was partially adherent to the vessel wall

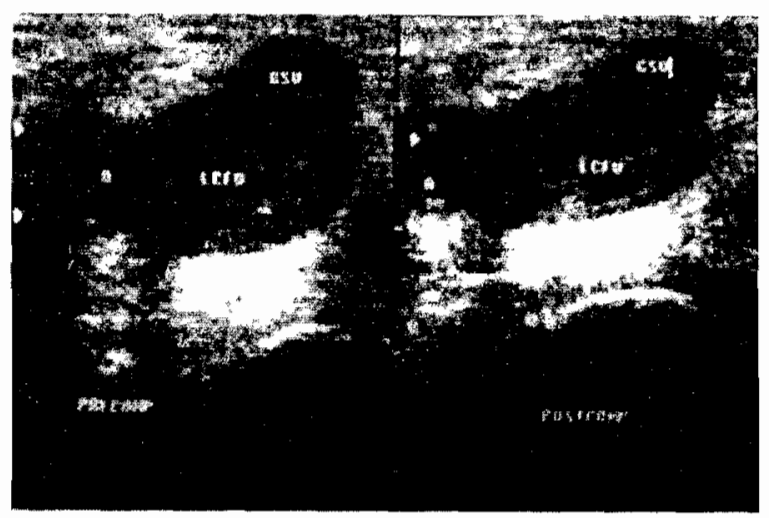

\section{Figure 2}

Transverse scans of the left common femoral vein in a cardiac patient who had left lower limb pain. There was an isoechoiec thrombus

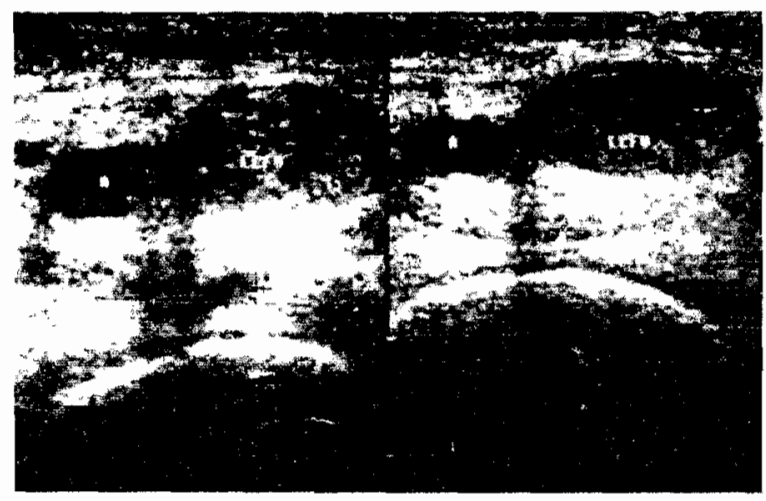

enlarged inguinal lymph nodes $(n=5)$, Cellulitis $(n=2)$, Baker's cyst $(n=1)$ and gastrocnemius tumour $(n=1)$. Thirty nine patients had normal findings.

\section{DISCUSSION}

Socio-demographic profiles and risk factors: The majority of the patients in this study and those who eventually had positive DVT studies were adults aged above 30 years. There were two age peaks at which DVT occurred. The highest peak between 31 and 40 years and another one at 61 to. 70 years. The two peaks probably represent the ages at which the significant risk factors for DVT in our setting are most common. These risk factors are immobility / prolonged bed rest, previous history of thromboembolism, major trauma, previous surgery, pregnancy and pueperium. Others are oral contraceptive use, obesity, malignancy and cardiac disease.

Immobility/prolonged bed rest, which was the most common risk factor observed in the study, plays a big role in the younger age group because this is the age group made morbid by trauma like road traffic accidents. At the same time this is the most active age group and this also increases that risk of trauma. After 60 years people tend to be less mobile therefore immobility still plays a big role in the aetiology of thrombosis in the 61 to 70 year age group. Pregnancy, oral contraceptive use and puerperium are the other risk factors seen in the young age group. Malignancy which is common in old age may also contribute to the aetiology of DVT 
in the 61 to 70 year age group. The incidence of DVT decreases after 70 years because few people in Uganda live beyond this age. The above age trend differs from what Anderson et al (3) in the Worcester DVT study observed where the incidence of thromboembolic disease steadly increased with age. They found that the annual incidence rates for DVT increased linearly from 17 per 100,000 between the ages of 40 to 49 to 232 per 100,000 between the ages of 70 and 79.

Venous stasis associated with bed rest and immobilisation is an important risk factor for venous thrombosis. A strong association ( $\mathrm{P}$-value $=0.004)$ was found between this factor and DVT. The greater influence of immobility is probably due to our hospital practices where early ambulation of patients is not emphasised and post-surgical sepsis, a common complication, leads to prolonged bed rest. Our patients also tend to stay in hospital for long because many of them come from far and therefore are kept in hospital till they attain complete recovery.

Among the patients, females were the majority accounting for $61.6 \%$ and males $39.4 \%$. This tallies with the findings of the September 2002 Uganda population and housing census where females accounted for $51.1 \%$ of the Ugandan population. However, this could also be because more females attend hospital than males in Uganda. They also have more conditions mimicking thromboembolism in presentation, like pregnancy and its related complication of pre-eclampsia (4).

Despite this, there was no difference in the gender incidence of DVT, which was 1:1. Given the fact that quite a number of risk factors are specific to females i.e. pregnancy, puerperium and oral contraceptive use, the similar sex incidence may be explained by the fact that in Uganda males are more exposed to trauma and therefore prolonged immobility and orthopaedic procedures, which are significant risk factors for DVT. This is because of the working habits of the Ugandan society whereby men are more involved in heavy work and travelling and therefore get exposed to road traffic accidents and injuries.

Most soldiers as well as builders are also men and are at risk of injury. This observation was reported by Kiguli-Malwadde et al (5). On the other hand most women in Uganda are full-time housewives and so do not travel much and do not do hard manual labour.
A previous history of thromboembolism, the second most common risk factor encountered had an odds ratio of $2.27(95 \% \mathrm{Cl}$ of $0.43-13.9)$. A similar finding was observed by Samama et al (6) in a community based epidemiological study of risk factors for DVT. They reported a relative risk of 7.9 conferred by a previous history of DVT (6). Chronic damage to venous endothelial linning, an aetiological factor of DVT, is a sequelae of venous thromboembolism. Collateral circulation which develop following chronic thrombosis cause sluggish blood flow further predisposing to recurrence of DVT. However it is important to note that US has limitations in the diagnosis of recurrent thrombosis. Heijboer et al (7) found normalisation of the test to take long after a first episode. Therefore some of the recurrences may not have been new thrombotic episodes, since most of the patients did not have previous imaging reports stating which veins were involved the first time.

Among the six patients who had had recent surgery, three $(50 \%)$ underwent orthopaedic operations involving the lower limb (hip surgery, internal fixation of a tibial fracture and a femoral fracture). Two of these (hip surgery and internal fixation of femoral fracture) were later found to have proximal DVT. This finding is not surprising since orthopaedic operations especially hip surgery has strongly been associated with lower limb thromboembolism. Coon (8) suggested a significant association between surgery especially orthopaedic operation and DVT. The mechanisms of activation of the coagulation system following trauma or surgery are incompletely understood but may include venous stasis, diminished fibrinolysis, the release or exposure of tissue factor and depletion of endogenous anticoagulants like antithrombin III. In addition the femoral vein in the operated limb may kink following hip surgery.

The odds ratio of pregnancy and oral contraceptive usage as risk factors were $1.29(95 \%$ Cl 0.39 to 22.78 ) and $4.03(95 \% \mathrm{Cl} 0.35$ to 105.07$)$ respectively. The wide confidence intervals are because of the small numbers of patients involved. Kierkegaard found a relatively low incidence of DVT in pregnancy occurring at a rate of 0.13 to 0.16 per thousand pregnancies (9).

The low incidence of oral contraceptive use as a risk factor in our setting may be explained by the low incidence of use of this method for family 
planning. The fact that it is used mainly by young females at which age there are very few confounders may also be responsible for the low incidence.

This observation is noted in the September 2000March 2001 Uganda Demographic and Health Survey which surveyed households and 7246 women aged 15 to 49 years. Contraceptive prevalence rate among currently married women aged 15 to 49 was $23 \%$ with prevalence of pill use being only $3 \%$. Only $26 \%$ of all women attending family planning clinics use oral contraceptives as a method of family planning. High pill use is also associated with young age. This shows that contraceptive pills are not used much in Uganda.

In previous studies done in Europe, the risk of thromboembolism increased three to four-fold higher in puerperium than in pregnancy (10). This was attributed to medical practices like operative (Caesarian or instrument) delivery, prescription of prolonged bed rest after delivery and use of oestrogens to suppress lactation. This is not evident in this study because Ugandan mothers are ambulant and start domestic work almost immediately after a normal delivery. There are none or very few cases of self prescribed operative deliveries or use of oestrogens to suppress lactation as occurs in developed countries.

Body mass index as a measure of obesity (BMI equal and above 30 ) had no significant association with DVT as only three out of eight obese patients had DVT. Obesity is a documented risk factor for DVT. However the proportion of obese people in the Ugandan populace is low as observed from this study population where only eight $(9.3 \%)$ out of eighty six patients were obese.

Malignancy and cardiac disease had no significant association with thromboembolic disease, but these fincings could be partly due to the small numbers of patients seen with these two risk factors. The low survival rates of patients with malignancy even with therapy in Uganda may account for this finding in that most of them do not live to develop thromboembolism. They usually present very late due to socio-economic problems, long distances from hospital and low doctor/patient ratio.

Clinical presentation: Limb swelling and local pain constituted the most common presenting symptoms. Thirty nine $(97.5 \%)$ and thirty five $(87.5 \%)$ out of forty of the thrombosed limbs had swelling and local pain respectively. This could be because pain is the main reason why patients usually present to hospital in Uganda. These were not useful symptoms as many patients without DVT also had similar symptoms. Forty three $(86.7 \%)$ and thirty two $(61.5 \%)$ respectively out of 52 limbs without DVT had these symptoms. Prior studies have shown pain and tenderness are not useful diagnostic signs. When five clinical studies were compared the sensitivity of calf pain varied from 66 to $91 \%$ and the specificity 3 to $87 \%$ (11).

Presence of pedal oedema was also unreliable sign of DVT although it had significant statistical association (P-value $=0.029$ ). Out of the 52 limbs with negative studies for DVT, $37(71.2 \%)$ presented with oedema implying a large number of conditions give oedema and therefore mimic DVT. The findings show that the chances of having a clot were markedly increased if the patient had unilateral leg oedema compared to those with bilateral leg and no oedema $(P$-value $=0.022)$.

Measurement of the difference in mid-calf circumference between the two limbs is a more useful sign compared with others, as the above results show. A difference of $2 \mathrm{~cm}$ and above highly suggests DVT. Homans' sign was also unreliable as it was positive in only $37.5 \%$ of those with DVT. A finding of a warm limb was a more reliable sign than a positive Homans' sign. Fourteen out of nineteen limbs with a raised temperature had thrombosis. The association was significant ( $P$-value $=0.03$ ).

Superficial venous dilatation, limb discolouration and the finding of a palpable cord were seen in a few cases of the study population $(2$, 12 and 3 respectively) and therefore it was difficult to make meaningful statistical deductions given the small sample size in the study. As regards limb discolouration, noticing it in our black population may be difficult, but this was partly overcome by comparing both limbs.

Wheeler HB, using impedance plethysmography also established that DVT cannot be diagnosed on the basis of history and physical examination, even in the high risk patients (2).

Sonographic findings: The-majority of deep venous thrombosis were found in the left leg $(60 \%)$. This is expected because of compression of the left iliac vein by the crossing right iliac artery at its origin from the aorta thereby reducing flow velocity and hence stasis in this vein. 
Most of the patients had proximal vein thrombosis. Isolated calf vein thrombosis was seen in only four cases. This could be due to the technical inadequacy of US in evaluating calf veins, because of their small size, anatomical variability and slower blood flow. US sensitivity in this case ranges from 73 to $87 \%$ (12-14).

The majority of the patients had extensive thrombosis. This implies they were presenting a little late for imaging, after thrombi had extended to involve several veins. This tallies closely with Makki et al (15) findings. Out of the 269 cases of acute DVT evaluated retrospectively, thrombosis extended from the common femoral to the popliteal vein in 207 $(77.7 \%)$ of cases, and isolated to the superficial femoral vein in $60(22.3 \%)$ of cases.

The late presentation of our patients for imaging was due to delay in referral, probably because of the lack of awareness of the people who handle these patients about the role of US as a quick and safe means of objective diagnosis of DVT. Pain which makes patients come to hospital, in our setting, is probably a late symptom of DVT. This could also account for late presentation.

Acute thrombus usually expands the vein. Ninety percent of the clots which were found in the common femoral veins had dilated the veins, suggesting a majority of the patients presented with acute thrombosis. Given the fact that the majority of the patients had extensive thrombosis, means there was rapid extension of thrombosis within the venous system.

Chronic thrombus on the other hand tends to narrow the vein as the clot retracts and the vein resumes its normal caliber, or contracts, due to damage of endothelial lining, with secondary scarring.

The majority of thrombi were partially adherent to the venous wall with a free floating component. Fresh or acute clot tends to be free floating in its early stages, and may be seen to waver within the vein with respiration or move within the vein during compression. This further confirms the fact that the majority of our patients had acute thrombosis. Most of the other diagnoses found at sonography (6/9) were pathological conditions in the inguinal region and popliteal fossa. These should be target areas meriting further evaluation, particularly when a study is negative for DVT. This observation does not differ from what James and Gary found (16). They prospectively evaluated 664 consecutive patients (886 limb examinations) with clinically suspected lower limb DVT using duplex Doppler venous examination (16). Of the 657 negative examinations in this study, 57 showed other positive diagnosis. Of the $57(74 \%),(42 / 57)$ of these diagnoses showed a lesion in the inguinal region or the popliteal fossa.

The remaining three out of nine patients with other diagnosis in our study had cellulites $(n=2)$ and a tumour of the gastrocnemius muscle $(n=1)$. This further demonstrates the weal th of information that can be obtained by sonography in evaluating lower limb symptoms. Thirty nine out of the forty eight patients who had no DVT in our study had normal findings at sonography.

In conclusion, the study results show that venous US is a very useful modality for assessing lower limb DVT even in a low resource country like Uganda. It demonstrates the wealth of information obtained from sonography.

\section{REFERENCES}

1. Hirsh J., Hull R.D. and Raskob G.E. Clinical features and diagnosis of venous thrombosis. J. Amer. Coll. Cardiol. 1986; 8: 1148-1278.

2. Wheeler H.B. Diagnosis of deep venous thrombosis; review of clinical evaluation and impedance plethsmography. Amer. J. Surg. 1985; 150 (suppl): 7-10.

3. Anderson F.A. Jr., Wheeler H.B., Goldberg R.J., et al. Physician practices in the prevention of various thromboembolism. Arch. Intern. Med. 1991; 115:591-595.

4. Sellman J.S., Russell L. and Hollman. Thromboembolism during pregnancy; risks, challenges, and recommendations. Postgraduate Med. 2000; 108: 1-11.

5. Kiguli-Malwadde E., Kawooya M.G., and Matovu D.I. The value of CT in the management of head trauma patients at Mulago Hospital. East and Central Afr. J. Surg. 1998; 4: 42-43

6. Samama M.M., Simmoneau G. and Wainstein J.P. Epidemiology of risk factors for deep venous thrombosis of the lower limb in community practice. Thromb. Haenost. 1993; 69: 797-800.

7. Heijboer H., Jongbloets L.M., Buller H.R. et al. Clinical utility of real time compression ultrasonography for diagnostic management of patients with recurrent venous thrombosis. Acta. Radiol. 1992; 33: 297-300.

8. Coon W.W. Epidemiology of venous thromboembolism. Ann. Surg. 1977; 149-151. 
9. Kierkegaard A. Incidence and diagnosis of deep vein thrombosis associated with pregnancy. Acta. Obstet. Gynecol. Scand. 1983; 62: 239-243.

10. Toglia M.R. and Weg J.G. Venous thromboembolism during pregnancy. Neto Engl. J. Med. 1996; 335: 108-114.

11. Leclerc J.R., Illescas F. and Jarzem P. Diagnosis of deep vein thrombosis. Venous Thromboembolic Disorders. Leclerc J.R (Ed). Lea and Febiger, Philadelphia. 1991; 303-345.

12. Habschied W., Hohmann M., Wilhelm T., et al. Real time ultrasound in the diagnosis of acute deep venous thrombosis of the lower extremity. Angiology. 1990; 41: 599-608.

13. Mitchell D.C., Grasty M.S., Stebbings W.S.L, et al. Comparison of duplex sonography and venography in diagnosis of deep vein thrombosis. Brit. J. Surg. 1991; 78: $732-739$.

14. Rose S.C., Zwebel W.J., Nelson B.D., et al. Symptomatic lower extremity deep vein thrombosis; accuracy, limitations and role of colour duplex flow imaging in diagnosis. Radiology. 1990; 175: 639-644.

15. Maki D.D., Kumar N., Nguyen B., et al. Distribution of thrombi in acute lower extremity deep venous thrombosis; implications of sonography and CT venography. Amer. J. Roetgen. 2000; 175: 1219-1301.

16. James P.B. and Gary E.C. Types, frequency and significance of alternative diagnoses found during duplex Doppler venous examinations of the lower extremities. J. Ultrasound Med. 1992.; 11: 85-89. 